\title{
Analyse De L'évolution Contemporaine De La Température De L'air Et Des Précipitations Sur Le Territoire Du Mali
}

\author{
Dr. Hamidou Diawara
}

Université des Sciences Sociales et de Gestion de Bamako (USSGB), Mali

Doi:10.19044/esj.2019.v15n2p154～URL:http://dx.doi.org/10.19044/esj.2019.v15n2p154

Résumé

Cette étude visait à analyser les variations décennales et à long termes de la température et des précipitations intervenues au Mali au cours des 80 dernières années du $\mathrm{XX}^{\mathrm{e}}$ siècle. Pour cette raison, une base de données de ces éléments sur 33 stations météorologiques à travers tout le pays a été conçue. La première étape a été la soumission des séries de données au contrôle de qualité via des tests statistiques. Ensuite elles ont été restaurées avec succès et utilisées pour l'estimation des normales climatiques avec la méthode des moyennes glissantes. La comparaison des cartes de répartition des précipitations révèle une diminution de celles-ci de $115 \mathrm{~mm}$ (soit de -18,3\%) au cours de la période de 1921 à 2000 , entraînant un déplacement des isohyètes vers le sud de $135 \mathrm{~km}$. Elle nous a permis de comprendre l'évolution de la température et des précipitations sur plusieurs décennies du siècle passé au Mali.

Mots-clés: Changement climatique, Normale climatique, Tests statistiques, Isohyètes, Moyenne glissante 


\title{
Analysis of the Contemporary Evolution of Air Temperature and Precipitation in the Territory of Mali
}

\author{
Dr. Hamidou Diawara
}

Université des Sciences Sociales et de Gestion de Bamako (USSGB), Mali

\begin{abstract}
This paper focuses on analyzing decadal and long-term changes in temperature and rainfall in Mali over the last 80 years of the $20^{\text {th }}$ century. In this study, a database of these elements on 33 weather stations across the country has been designed. The first step was the submission of data sets to quality control via statistical tests. Afterwards, they were successfully restored and used to estimate climatic normals with the sliding average method. The comparison of rainfall distribution maps shows a decrease of $115 \mathrm{~mm}$ (or $18,3 \%$ ) during the period from 1921 to 2000 , resulting in a displacement of isohyets to the south of $135 \mathrm{~km}$. This allowed us to understand the evolution of temperature and rainfall over several decades of the last century in Mali.
\end{abstract}

Keywords: Climate change, Climate normal, Statistical tests, Isohyets, Moving average

\section{Introduction}

Dans la plupart des pays en développement l'agriculture est le plus important secteur de l'économie. Selon le rapport de la Conférence des Nations Unies pour le Commerce et le Développement (CNUCED, 2010), elle fournit 20\% à 60\% du Produit Intérieur Brut (PIB), 65\% des emplois de la population active selon le pays et les moyens de subsistance pour environ 2,6 milliards de personnes dans le monde.

Dans ce sens, les changements climatiques pourraient causer des dommages irréversibles à la base des ressources naturelles dont dépend l'agriculture, avec toutes les conséquences graves qui en découlent pour la sécurité alimentaire dans les pays en développement. Le réchauffement climatique en menaçant la production agricole et le commerce des produits agricoles augmente donc le risque de la malnutrition et de la faim extrême dans le monde.

Les estimations de la CNUCED (CNUCED, 2010) pour 2080 indiquent que, dans les régions en développement les plus touchées par les 
changements climatiques, la productivité agricole diminuera d'environ $15 \%$ à $30 \%$, voire de $50 \%$ dans certains pays de ces régions.

Ainsi, il apparaît clairement l'importance et la pertinence de l'évaluation du changement climatique dans le monde, en particulier dans les pays de l'Afrique, dont le Mali, qui sont parmi les plus vulnérables. En effet, depuis 2010 le Mali est classé parmi les pays à vulnérabilité climatique sévère, ce qui devrait passer à aigue à l'horizon 2030 (Fundation DARA International, 2010).

Les études menées sur les changements climatiques dans les pays de l'Afrique (Kouassi et al., 2010; Roudier, 2012) traitent pour la plupart la question de manière régionale. Pour certaines, elles ne s'intéressent pas assez à la période avant le milieu du $\mathrm{XX}^{\mathrm{e}}$ siècle car elles considèrent la période 19511980 comme la référence de l'Organisation Météorologique Mondiale (OMM) (Ouedraogo, 2001). Pourtant, même celles qui abordent le problème à une échelle spatiale réduite, notamment l'étude de Briquet et al. (1996), n'accordent pas souvent assez d'attention à l'analyse statistique préalable sur la qualité les données climatiques avant leur interprétation, ce qui peut conduire à des conclusions discutables en cas de valeurs aberrantes. Par contre, Paturel et al. (1998), en analysant les séries pluviométriques de longue durée d'Afrique de l'Ouest et Centrale non sahélienne s'étaient intéressés à la vérification de leur qualité, ce qui leur a permis d'identifier l'alternance de trois périodes homogènes (déficitaire-excédentaire-déficitaire) de la pluviométrie entre 1936 et 1990. Mais là aussi les données utilisées sont assez vieillissantes. Ce constat est commun à toutes les autres études citées plus haut car elles ne se sont toutes intéressées, comme L'hôte et al. (1996), lors de leur classification des types de climats en Afrique noire à l'Ouest du Congo, qu'aux données météorologiques du milieu à la fin du siècle précédent.

À cet égard, cette étude s'inscrit donc dans la logique globale des précédentes, mais en mettant l'accent sur l'échelle spatiale nationale et en utilisant des données relativement plus récentes afin de comprendre la situation de la dynamique plus récente de la température et des précipitations au Mali. Elle pourra sûrement servir de base pour des prévisions de la situation agricole future, fortement dépendante du climat, à cours et à moyen termes. Cette étude vise donc à analyser les variations décennales et à long termes de la température et des précipitations intervenues au Mali au cours des 80 dernières années du $\mathrm{XX}^{\mathrm{e}}$ siècle.

\section{Matériels et méthodes}

\section{Présentation de la zone d'étude}

L'étude a été réalisée sur l'ensemble du territoire du Mali. Le pays, situé au cœur de l'Afrique de l'Ouest, est caractérisé par un climat chaud avec l'alternance d'une saison sèche et d'une saison des pluies. La durée de ces 
deux saisons varie inversement du sud au nord. La première est courte au sud et longue au nord. Le cumul pluviométrique annuel sur le territoire varie de moins de $200 \mathrm{~mm}$ à plus de $1000 \mathrm{~mm}$. Selon la quantité de pluie reçue par an et la durée de la saison des pluies, le territoire du Mali est habituellement divisé en quatre zones agroclimatiques (DNM, 2007); il s'agit, du sud au nord, de:

- la zone Soudano-guinéenne (ou pré-guinéenne) avec une saison des pluies qui dure plus de 6 mois et une pluviométrie annuelle depassant 1000 $\mathrm{mm}$;

- la zone Soudanienne dont la saison des pluies dure environ 5 mois et le cumul annuel des pluies se situe entre $600 \mathrm{~mm}$ et $1000 \mathrm{~mm}$;

- la zonne Sahélienne avec une pluviometrie de $200 \mathrm{~mm}$ à $600 \mathrm{~mm}$ en 3-4 mois;

- la zone Saharienne dont la pluviométrie annuelle est inférieure à 200 mm et la saison des pluies ne dure que 2 mois.

\section{Collecte des données}

Pour mener cette étude nous avons eu recours aux données mensuelles des températures de l'air et de la pluviométrie au Mali. Ces données ont été mesurées sur 33 stations météorologiques. La répartition sur le territoire des stations est présentée sur la Figure 1.

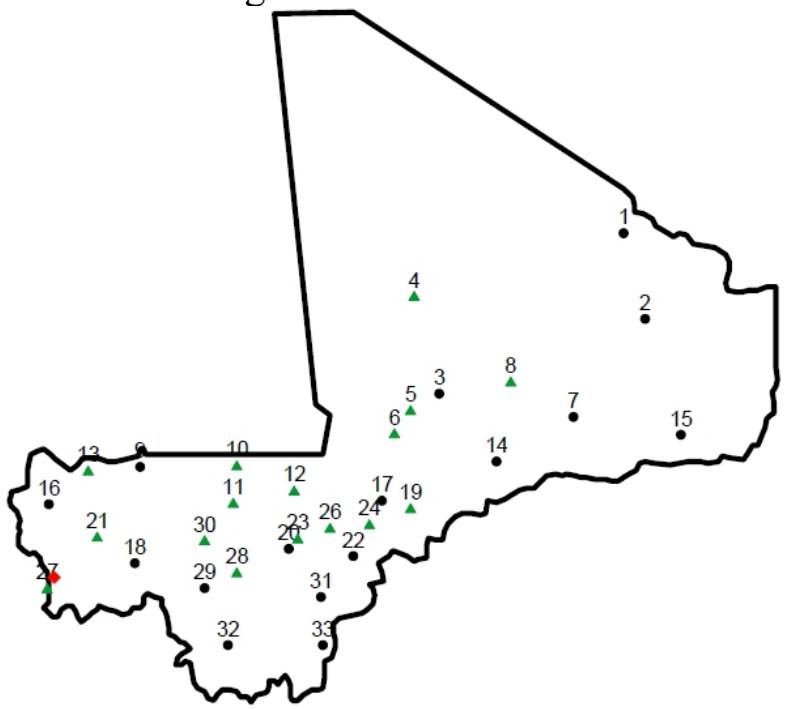

Figure 1. Répartition territoriale des stations météorologiques.

- Stations d'observations des températures et des précipitations ( $\mathrm{T}$ et $\mathrm{P})$.

$\Delta \quad$ Stations d'observations des précipitations $(\mathrm{P})$.

Stations d'observations des températures $(\mathrm{T})$.

La carte permet de constater que la réparation des stations météorologiques est dense au sud et au centre du pays, et de faible à inexistante dans la partie nord. La qualité des séries de données varie d'une station à une 
autre. Il y’a un écart plus ou moins significatif entre les années de début et de fin des observations des températures et des précipitations des stations. Les caractéristiques de la base de données météorologiques figurent dans le Tableau 1.

Tableau 1. Les caractéristiques de la base de données météorologiques

\begin{tabular}{|c|c|c|c|c|c|}
\hline \multicolumn{3}{|c|}{ Température } & \multicolumn{3}{c|}{ Précipitations } \\
\hline $\begin{array}{c}\text { Nbre de } \\
\text { stations }\end{array}$ & $\begin{array}{c}\text { Période } \\
\text { maximale }\end{array}$ & Durée moyenne & $\begin{array}{c}\text { Nbre de } \\
\text { stations }\end{array}$ & $\begin{array}{c}\text { Période } \\
\text { maximale }\end{array}$ & $\begin{array}{c}\text { Durée } \\
\text { moyenne }\end{array}$ \\
\hline 17 & $1896-2011$ & 68,1 & 32 & $1895-2011$ & 83,5 \\
\hline
\end{tabular}

La période d'observation de la plus longue série est de 1896-2011 pour la température et 1895-2011 pour les précipitations. La durée moyenne des observations des températures est de 68,1 ans tandis que celle des observations des précipitations est de 83,5 ans. Pour faciliter le traitement des données dans des logiciels appropriés, un code de cinq chiffres, différent de celui de l'Organisation Météorologique Mondiale (OMM), a été attribué à chacune des stations.

\section{Contrôle de la qualité et correction des données collectées}

La procédure contrôle-qualité et correction des séries de données a été effectuée en quelques étapes (Lobanov et al., 2011) :

- L'analyse des valeurs extrémales susceptibles d'impacter l'homogénéité des séries de données a été effectuée grâce aux tests de Dixon et de Grubbs (tests pour l'identification et l'élimination des valeurs extrémales aberrantes). Ces tests ont été effectués au niveau de confiance $95 \%$.

- $\quad$ La stationnarité statistique de la normale et de la dispersion des séries a été analysée respectivement avec les tests de Student et de Fisher (test de Fisher-Snedecor) au même niveau de confiance.

- $\quad$ La correction et l'extrapolation des séries de données météorologiques ont été effectuées sur la base de l'analogie des processus naturels au niveau des stations. Autrement dit, elles ont été effectuées sur la base de l'analyse corrélationnelle des séries des différentes stations avec la construction d'équations de régression linéaire multiple entre celles-ci. Le degré de signification de la liaison entre les variables est évalué par le coefficient de corrélation de Pearson $(r)$ dont la valeur absolue varie de 0 (absence totale de liaison) à 1 (une très forte liaison).

Tous les calculs des coefficients de corrélation, des droites de régression linéaire simple et multiple et des interpolations et/ou des extrapolations sont réalisés grâce au logiciel «Hydroratchoti» (logiciel d'analyse quantitative). 
Le choix des stations analogues (avec des séries $x_{1}, x_{2}, x_{3}, \ldots$ ), dont les données ont été utilisées comme des variables indépendantes dans les équations de régression, a été fait sur la base de :

- la proximité géographique avec la station $(y)$ dont la série est à corriger (à restaurer);

- la variation synchronisée des séries des stations ( $y$ et $x_{1}, x_{2}, x_{3}, \ldots$ ) ;

- la longueur des séries des stations analogues supérieure à celle de la série de la station à restaurer ;

- le coefficient de corrélation entre les séries supérieur ou égal à 0,80 .

\section{Évaluation des caractéristiques climatiques et leurs spatialisations}

Pour le calcul des valeurs normales climatiques des températures de l'air et des précipitations nous avons appliqué les normes établies en la matière par l'OMM (OMM, 2011); c'est-à-dire, en divisant la série en plusieurs périodes de 30 ans et en calculant la moyenne pour chacune d'elles. En plus, pour cause des changements climatiques, il est aussi recommandé la vérification des normales tous les 5 ans ou 10 ans (Kouassi et al., 2010). Cela a conduit au schéma suivant de calcul des moyennes glissantes :

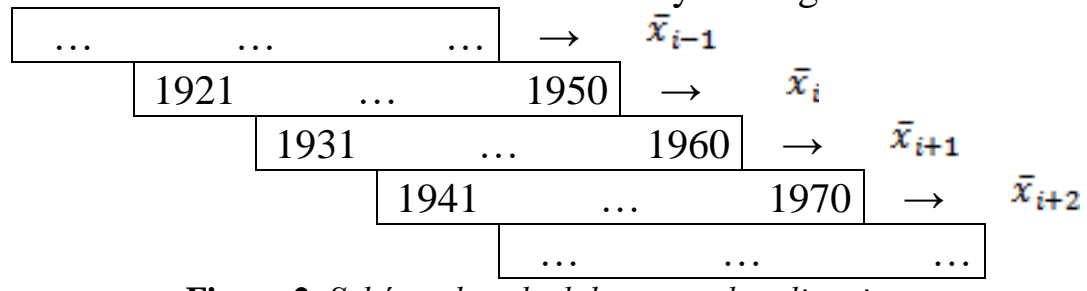

Figure 2. Schéma de calcul des normales climatiques

Ainsi, les normales des températures moyennes annuelles et des cumuls annuels des précipitations, pour chacune des périodes de 30 ans, ont été calculées par la formule suivante:

$$
\bar{x}=\frac{\sum_{i=1}^{n} x_{i}}{n},
$$

où $n=30$ ans.

L'analyse de la variation de ces normales a été faite sur la base des écarts relatifs entre celles-ci pour deux périodes consécutives. Ces écarts sont calculés par la formule ci-après:

$$
\Delta \bar{x} \%=\frac{\bar{x}_{i+1}-\overline{x_{i}}}{\overline{x_{i}}} 100
$$

La valeur et le signe de $\Delta x \%$ nous indiqueront le degré et la nature des variations de la normale. Lorsque $\Delta x \%$ est positif, alors il y a augmentation de la normale, et lorsque $\Delta x \%$ est négatif, c'est le cas de la diminution de la normale. 
Enfin la cartographie des caractéristiques calculées a été réalisée avec l'aide de ArcView 3.2 pour l'extraction de la carte basique et de Surfer 8 pour les interpolations spatiales. La méthode d'interpolation utilisée pour la construction des isolignes (isothermes et isohyètes) est celle de Krigeage.

\section{Résultats}

Les principaux résultats obtenus de l'étude sont relatifs aux différents tests statistiques auxquels ont été soumises les séries de données, aux caractéristiques statistiques obtenues et à la spatialisation et l'analyse de celles-ci.

\section{Qualité des séries de données et restaurations de celles-ci}

Les résultats du contrôle-qualité (des tests statistiques de Fisher $F$ et Student $t$ ) et de la restauration (et du prolongement) des séries de données des stations sont récapitulées dans le Tableau 2.

Tableau 2. Résultats des tests statistiques et de la restauration des séries de données de la température de l'air et des précipitations

a) Test de Fisher

\begin{tabular}{|r|c|r|r|r|r|}
\cline { 2 - 6 } \multicolumn{2}{c|}{} & \multicolumn{2}{c|}{ Température } & \multicolumn{2}{c|}{ Précipitations } \\
\hline \multicolumn{2}{|c|}{ Statistique de Fisher } & \multicolumn{2}{c|}{ n } & \multicolumn{1}{c|}{ n } & \multicolumn{1}{c|}{} \\
\hline \multirow{2}{*}{$F$} & + & 14 & 82,4 & 24 & 75,0 \\
\cline { 2 - 7 } & - & 3 & 17,6 & 8 & 25,0 \\
\hline \multicolumn{2}{|c|}{ Total } & 17 & 100,0 & 32 & 100,0 \\
\hline
\end{tabular}

b) Test de Student

\begin{tabular}{|c|c|c|c|c|c|}
\hline & & \multicolumn{2}{|c|}{ Température } & \multicolumn{2}{|c|}{ Précipitations } \\
\hline \multicolumn{2}{|c|}{ Statistique de Student } & $\mathrm{n}$ & $\%$ & $\mathrm{n}$ & $\%$ \\
\hline \multirow{2}{*}{$t$} & + & 16 & 94,1 & 10 & 31,2 \\
\hline & - & 1 & 5,9 & 22 & 68,8 \\
\hline \multicolumn{2}{|c|}{ Total } & 17 & 100,0 & 32 & 100,0 \\
\hline
\end{tabular}

Significations : "+ »= stable (homogène), « - »= instable (non homogène) ; $n=$ nombre.

c) Caractéristiques des données avant et après restauration

\begin{tabular}{|c|r|r|r|r|r|r|}
\cline { 2 - 7 } \multicolumn{1}{c|}{} & \multicolumn{3}{c|}{ Température } & \multicolumn{3}{c|}{ Précipitations } \\
\hline Caractéristique & \multicolumn{1}{c|}{ Avant } & \multicolumn{1}{c|}{ Après } & \multicolumn{1}{c|}{ Ec.\% } & \multicolumn{1}{c|}{ Avant } & \multicolumn{1}{c|}{ Après } & \multicolumn{1}{c|}{ Ec.\% } \\
\hline Taille de la série (an) & 68,1 & 85,6 & 25,7 & 83,5 & 103,6 & 24,1 \\
\hline Valeur moyenne & 28,4 & 28,6 & 0,7 & 580 & 565 & $-2,6$ \\
\hline
\end{tabular}

Significations : Avant $=$ Avant restauration; Après $=$ Après restauration; Ec. $\%=$ écart relatif en \% de la moyenne/taille de la série après restauration par rapport à sa moyenne/taille avant restauration.

L'observation des Tableaux a) et b) montre que 8 des séries de précipitations, soit $25,0 \%$, se sont avérées instables selon le test de Fisher. Ce qui conclue à une grande fluctuation des cumuls pluviométriques annuels sur 
le court terme. Par ailleurs, 22 séries des précipitations (soit 68,8\%) sont instables par la moyenne suite au test de Student.

Quant à la température, elle s'est avérée plus stable dans le temps que la pluviométrie. En effet, seulement 3 séries et 1 série (soit 17,6\% et 5,9\%) des 17 séries de températures sont instables respectivement selon la variance et la moyenne.

La restauration (voir Tableau c) a permis de rallonger les séries des précipitations de 24,1\% en moyenne sur le territoire (de 83,5 ans à 103,6 ans). Pour les séries de la température, elle a permis un ralongement de leur longueur moyenne de 17,5 ans. Le meilleur critère de vérification de la fiabilité de la restauration est la stabilité de la moyenne des séries avant et après restauration. Ainsi nous constatons que celle-ci n'a varié en moyenne sur l'ensemble des stations utilisées que de $-2,0 \%$ pour les précipitations et de $0,7 \%$ pour la température.

\section{Valeurs normales (climatiques) des éléments sur différentes périodes}

Bien que la durée des séries de données soit très longue pour certaines stations, les normales, par la méthode de la moyenne glissante, ont été calculées pour la période d'observations communes à toutes les stations pour chaque élément climatique (température et précipitations), ce qui est obligatoire lorsqu'on veut comparer et cartographier les caractéristiques statistiques des différentes stations. C'est ainsi que la période de 1941 à 2000 (60 ans) a été considérée pour la température et la période de 1921 à 2000 (80 ans), pour les précipitations.

Les normales climatiques sur des périodes de 30 ans avec un pas de 10 ans ont été calculées. Les écarts relatifs entres les normales consécutives ont également été calculés. Ces résultats sont montrés dans le Tableau 3 pour la température et le Tableau 4 pour les précipitations.

Tableau 3. Les caractéristiques des valeurs normales de la température moyenne annuelle et les écarts relatifs entres celles-ci

\begin{tabular}{|c|c|c|c|c|c|c|c|}
\hline \multirow{2}{*}{$\begin{array}{c}\text { Caractéristique } \\
\text { statistique } \\
\text { de la distribution } \\
\text { spatiale }\end{array}$} & \multicolumn{4}{|c|}{$\begin{array}{l}\text { Température normale sur la } \\
\text { période, en }{ }^{\circ} \mathrm{C}\end{array}$} & \multicolumn{3}{|c|}{$\begin{array}{c}\text { Écarts relatifs entre les normales } \\
\text { successives, en \% }\end{array}$} \\
\hline & $\begin{array}{c}1941 \\
- \\
1970 \\
\end{array}$ & $\begin{array}{c}1951 \\
- \\
1980 \\
\end{array}$ & $\begin{array}{c}1961 \\
- \\
1990 \\
\end{array}$ & $\begin{array}{c}1971 \\
- \\
2000 \\
\end{array}$ & $\Delta_{1}$ & $\Delta_{2}$ & $\Delta_{3}$ \\
\hline Minimum (min) & 27,0 & 26,9 & 26,8 & 26,8 & $-0,37$ & $-0,37$ & 0,00 \\
\hline Moyenne (moy) & 28,4 & 28,3 & 28,4 & 28,6 & $-0,35$ & 0,23 & 0,58 \\
\hline Maximum $(\max )$ & 30,1 & 30,1 & 30,3 & 30,7 & 0,00 & 0,66 & 1,32 \\
\hline Écart-type $(\sigma)$ & 0,92 & 0,95 & 1,00 & 1,09 & 3,55 & 4,48 & 9,22 \\
\hline $\begin{array}{l}\text { Coef. de variation } \\
(\mathrm{Cv} \%)\end{array}$ & 3,24 & 3,36 & 3,50 & 3,81 & 3,91 & 4,24 & 8,59 \\
\hline
\end{tabular}

En analysant les écarts relatifs nous pouvons dire que la période de 1951-1980 par rapport à celle de 1941-1970 a été caractérisée par un léger 
refroidissement sur le territoire dû au cycle naturel de la température, car la température moyenne y a connu une diminution de $0,35 \%$ environ. Cependant, depuis cette période, la température n'a cessé de croître faiblement pour la maximale et la moyenne qui a augmenté de 0,23\% entre 1961 et 1990 puis de $0,58 \%$ entre 1971 et 2000 . Au même moment, la minimale évoluait dans le sens inverse. De 1941 à 2000, les variations des températures (minimale, moyenne et maximale) sont très légères, n'atteignant pas $1{ }^{\circ} \mathrm{C}$.

Pour les précipitations, les résultats sont présentés dans le tableau suivant:

Tableau 4. Les caractéristiques des valeurs normales des précipitations annuelles et les écarts relatifs entres celles-ci

\begin{tabular}{|c|c|c|c|c|c|c|c|c|c|c|c|}
\hline \multirow[b]{2}{*}{$\begin{array}{l}\text { Caractéristique } \\
\text { statistique de la } \\
\text { distribution } \\
\text { spatiale }\end{array}$} & \multicolumn{6}{|c|}{$\begin{array}{l}\text { Précipitations normales sur la } \\
\text { période, en } \mathrm{mm}\end{array}$} & \multicolumn{5}{|c|}{$\begin{array}{l}\text { Écarts relatifs entre les normales } \\
\text { successives, en \% }\end{array}$} \\
\hline & $\begin{array}{c}192 \\
1 \\
- \\
195 \\
0\end{array}$ & $\begin{array}{c}1931 \\
- \\
1960\end{array}$ & $\begin{array}{c}194 \\
1 \\
- \\
197 \\
0\end{array}$ & $\begin{array}{c}195 \\
1 \\
- \\
198 \\
0\end{array}$ & $\begin{array}{c}196 \\
1 \\
- \\
199 \\
0\end{array}$ & $\begin{array}{c}197 \\
1 \\
- \\
200 \\
0\end{array}$ & $\Delta_{1}$ & $\Delta_{2}$ & $\Delta_{3}$ & $\Delta_{4}$ & $\Delta_{5}$ \\
\hline Minimum (min) & 44 & 35 & 35 & 34 & 36 & 32 & -20 & -17 & -12 & -28 & -11 \\
\hline Moyenne (moy) & 618 & 636 & 622 & 593 & 522 & $\overline{503}$ & 3 & -2 & -5 & -13 & -4 \\
\hline Maximum (max) & $\begin{array}{r}140 \\
6\end{array}$ & 1326 & $\begin{array}{r}133 \\
2\end{array}$ & $\begin{array}{r}128 \\
5\end{array}$ & $\begin{array}{r}114 \\
1\end{array}$ & $\begin{array}{r}110 \\
1\end{array}$ & 14 & 7 & 0 & 6 & 4 \\
\hline Écart-type $(\sigma)$ & $\begin{array}{r}364, \\
2\end{array}$ & $\begin{array}{r}368, \\
2\end{array}$ & $\begin{array}{r}360 \\
, 0 \\
\end{array}$ & $\begin{array}{r}346 \\
, 9 \\
\end{array}$ & $\begin{array}{r}321 \\
, 3 \\
\end{array}$ & $\begin{array}{r}311, \\
5\end{array}$ & 5,6 & 4,7 & 2,9 & 6,0 & 3,5 \\
\hline $\begin{array}{l}\text { Coef. de variation } \\
(\mathrm{Cv} \%)\end{array}$ & 59,0 & 57,9 & $\begin{array}{r}57, \\
9\end{array}$ & $\begin{array}{r}58, \\
5\end{array}$ & $\begin{array}{r}61, \\
5\end{array}$ & 62,0 & 205,9 & $-195,2$ & $-59,7$ & $-45,1$ & $-94,5$ \\
\hline
\end{tabular}

Ici, de la période 1921-1950 à la période 1931-1960, la normale des précipitations a augmenté sur $84,3 \%$ des 32 stations etudiées, mais en moyenne, sur tout le territoire, elle a diminué de $2,71 \%$ entre ces deux périodes. Enfin, pendant toutes les autres périodes jusqu'à 2000, nous constatons que les pluies n'ont cessé de diminuer sur l'ensemble du territoire du Mali (à l'image de la station de Sikasso dont la dynamique est illustrée sur la figure suivante), et en moyenne cela représente $18,6 \%$.

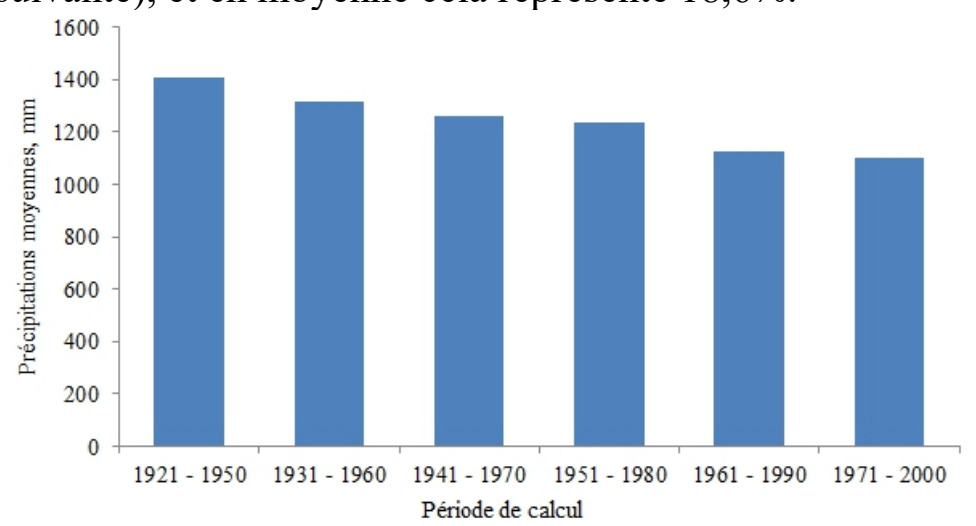

Figure 3. Dynamique de la normale des précipitations à Sikasso de 1921 à 2000. 
Le cas de Sikasso est important du fait que c'est la région la plus au sud du Mali, dans la partie du territoire qui reçoit plus de pluies que toutes les autres. Ici on constate qu'il pleuvait jusqu'à $1400 \mathrm{~mm}$ pendant la première moitié du $\mathrm{XX}^{\mathrm{e}}$ siècle, mais depuis les pluies ont diminué progressivement d'année en année. Et en 2000, la normale n'était plus que de $1100 \mathrm{~mm}$.

\section{Répartitions spatiales des normales climatiques}

Pour des besoins de visualisation et de comparaison spatio-temporelle de la distribution sur le territoire des normales de la température moyenne annuelle et des précipitations, les cartes ont été élaborées. Les résultats de la spatialisation, pour différentes périodes, des caractéristiques statistiques de ces éléments du climat (températures et précipitations) sont sur les figures suivantes :

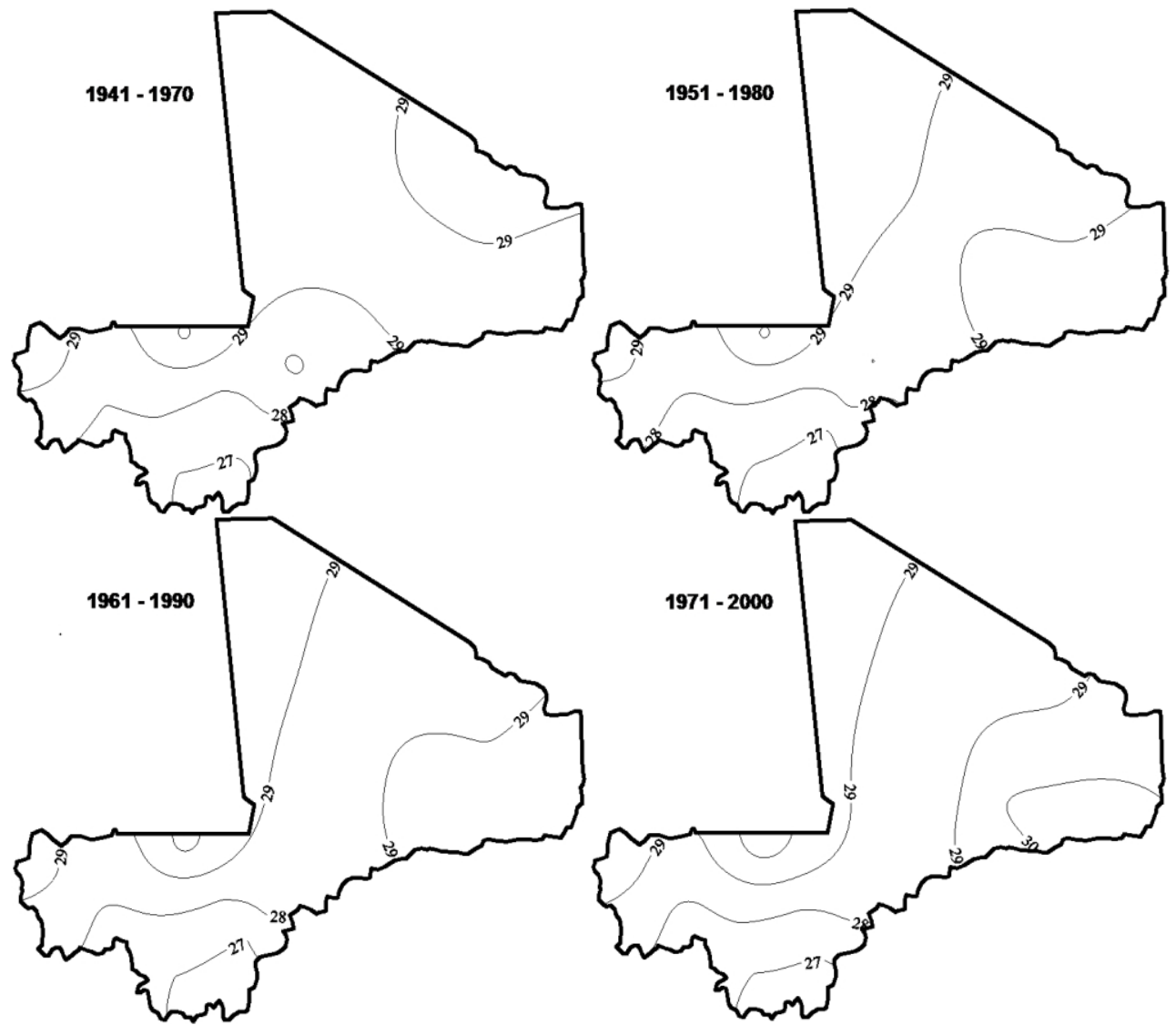

Figure 4. Distribution spatiale des normales de la température annuelle sur le territoire du Mali.

Ces cartes permettent de comprendre que la distribution de la température normale sur le territoire a un peu varié entre 1941-1970 et 19511980. Sur la durée, les températures ont subi une augmentation, mais pas équivalente dans toutes les localités. En effet, dans la partie sud du pays, la 
normale de la température a augmenté de $0,10^{\circ} \mathrm{C}$ à $0,20^{\circ} \mathrm{C}$ entre $1941-1970$ et 1971-2000. Sur la même période au nord, où il faisait déjà plus chaud, l'augmentation se situe entre $0,50{ }^{\circ} \mathrm{C}$ et $0,90{ }^{\circ} \mathrm{C}$.

Les distributions des précipitations quant à elles sont présentées sur les figures suivantes:

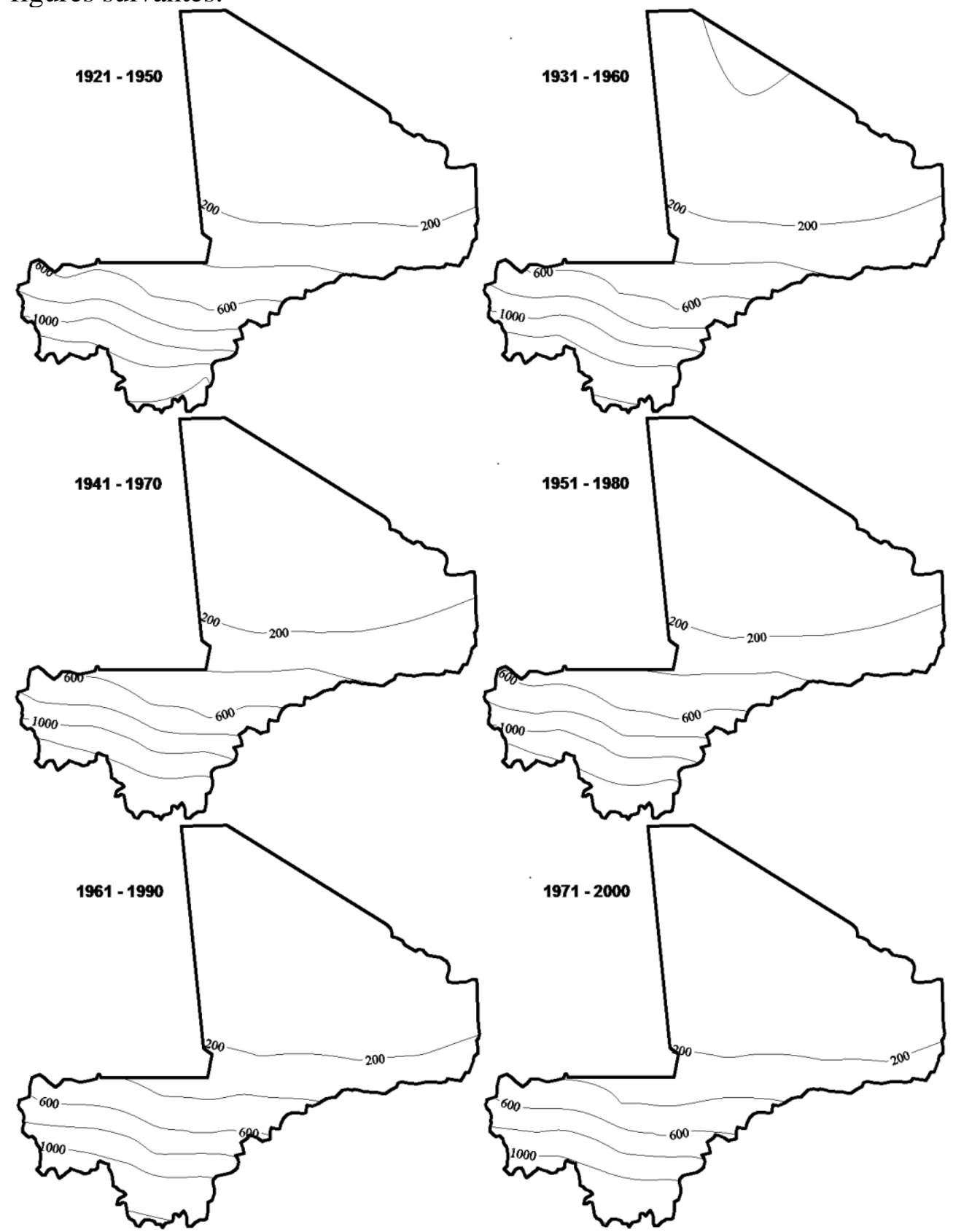

Figure 5. Distribution spatiale des normales des précipitations annuelles sur le territoire du Mali. 
À partir de ces cartes, nous constatons une variation de la normale des précipitations annuelles. On note une légère augmentation (déplacement des isohyètes vers le nord) de $2,9 \%$ de la normale des pluies sur le territoire entre 1921-1950 et 1931-1960, et depuis la pluviométrie n'a cessé de diminuer et les isohyètes de reculer progressivement vers le sud. En moyenne sur le territoire, elle a varié de $636 \mathrm{~mm} / \mathrm{an}$ entre 1931-1960 à $503 \mathrm{~mm} / \mathrm{an}$ entre 19712000 , soit une diminution de $20,9 \%$, conduisant à un recul des isohyètes de $135 \mathrm{~km}$ vers le sud.Cependant, rien que pour la seule décennie de 1981 à 1990, nous constatons la diminution de la pluie de $71 \mathrm{~mm}$, représentant 13,3\% (la plus grande variation de celle-ci en une décennie depuis 1921). Les isohyètes $1400 \mathrm{~mm}$ et $1200 \mathrm{~mm}$ ont disparu du territoire du Mali respectivement de 1980 à 1990 et de 1990 à 2000.

\section{Discussion}

L'analyse des résultats des tests statistiques de Fisher et de Student permet de dire que la dynamique de la température annuelle est globalement plus stable que celle des précipitations. On constate que l'homogénéité des séries de températures est mise en cause dans 5,9\% des cas (par les moyennes) et $17,6 \%$ (par les variances). Par contre pour les séries des précipitations, cela correspond à 22 séries sur 32 (soit 68,8\%) non homogènes par les moyennes et 8 séries (soit $25,0 \%$ ) non homogènes par les variances. Ces conclusions confirment l'instabilité des normales climatiques dans le temps depuis 1880 (GIEC, 2014) en générale, et le rythme accéléré de la variation des précipitations en Afrique de l'Ouest en particulier, déjà constatée par Paturel et al. (1998).

Les séries des données ont été restaurées et ralongées avec une efficacité moyenne de $29,7 \%$ pour la température et de $33,1 \%$ pour les précipitations. Les moyennes, avant et après les restaurations, des séries de températures et des précipitations sont restées presque constantes (une variation de moins de 5\% au niveau de la plupart des stations concernées). Cela nous a permis d'avoir des séries longues de données sans interruptions d'au moins 60 ans (de 1941 à 2000) pour la température et 80 ans (de 1921 à 2000) pour les précipitations.

Ensuite, l'analyse des Tableaux 4 et 5 permet d'affirmer que la normale des températures moyennes annuelles n'a pas varié de manière significative sur le territoire car sa variation est seulement de l'ordre de $+0,2^{\circ} \mathrm{C}$ (soit moins que $1 \%$ ) sur la période de 1941 à 2000. Mais pour les précipitations, il y a eu une diminution continue de la normale pour un total de $115 \mathrm{~mm}$, représentant $-18,6 \%$ de 1921 à 2000. La plus remarquable diminution de la pluie moyenne annuelle sur le territoire est intervenue entre 1980 et 1990, soit $70 \mathrm{~mm}$ ou $13,3 \%$ de pluie de moins par rapport à la normale de la période 1951-1980. 
Enfin, 1'analyse des cartes des températures normales permet de constater que la répartition de celle-ci n'a presque pas subi de modification et que le maximum $\left(30^{\circ} \mathrm{C}-31^{\circ} \mathrm{C}\right)$ intervient pratiquement toujours dans deux parties du pays: dans le nord-est (au sud du Sahara et du Sahel) et à l'est ; les noyaux de chaleur sont donc restés stables dans le temps. Par contre, les cartes des précipitations montrent une dynamique intense. On constate partout une diminution des pluies, ce qui confirme les tendances remarquées dans les études préalablement réalisées (Roudier \& Quirion, 2009). Cette diminution entre 1931-1960 et 1971-2000 s'estime à 20,9\% pour tout le Mali, conduisant à un recul des isohyètes vers le sud de $135 \mathrm{~km}$ entre 1921 et 2000 et de $90 \mathrm{~km}$ pour la seule période de 1980 à 1990. Par conséquent, les isohyètes $1400 \mathrm{~mm}$ et $1200 \mathrm{~mm}$, auparavant localisées dans le sud du pays, ont définitivement disparu du territoire du Mali respectivement entre 1980 à 1990 et entre 1990 à 2000. Beaucoup d'études régionales antérieures réalisées sur cette problématique, notamment celles de Le Barbe et al. (2001); Nicholson et al. (2007) avaient déjà permis de comprendre que depuis quelques décennies en Afrique de l'Ouest les isohyètes ont subi un glissement vers le sud. Par la même occasion, ils affirmaient que les précipitations ont en moyenne baissé de 15 à 30\% au Sahel. Selon le premier le déficit pluviométrique en Afrique de l'Ouest equivaut à 180-200 mm. Mais dans cette étude, les isohyètes occupent une position légèrement plus au nord comparativement à celles obtenues par Ouedraogo en 2001 pour la période de 1951 à 1997.

\section{Conclusion}

Les températures annuelles et les précipitations au Mali ont subi beaucoup de fluctuations au cours des 80 dernières années du siècle passé. En effet, les tests statistiques ont montré que respectivement $17,6 \%$ et $25,0 \%$ des séries de température et des précipitations sont instables par la variance. Par contre, par la moyenne, la température s'est avérée de loin plus stable que les précipitations car les taux d'instabilité pour les deux éléments sont respectivement $5,9 \%$ et $68,8 \%$. L'analyse de la dynamique spatio-temporelle de la température a permis de constater que la moyenne de celle-ci n'a pas cessé de croître depuis la période 1951-1980 jusqu'à la période 1971-2000. $\mathrm{Au}$ même moment, pendant que la maximale évoluait dans le sens de l'augmentation, la minimale évoluait dans le sens inverse. La normale de la température a beaucoup plus augmenté au nord qu'au sud du pays. Quant aux précipitations, elles ont diminué partout sur le territoire. Cette diminution des pluies entre 1931-1960 et 1971-2000 s'estime à 20,9\% pour tout le Mali. Les isohyètes ont reculé vers le sud de $135 \mathrm{~km}$ entre 1921 et 2000 , et de $90 \mathrm{~km}$ pour la seule période de 1980 à 1990. Par conséquent, les isohyètes $1400 \mathrm{~mm}$ et $1200 \mathrm{~mm}$, avant localisées dans le sud du pays, ont définitivement disparu du territoire du Mali respectivement entre 1980 à 1990 et entre 1990 à 2000. 
À partir de ce qui vient d'être dit, il est possible d' affirmer que la compréhension des dynamiques de la température et des précipitations au Mali est une étape importante dans le processus d'adaptation des secteurs de production du pays aux effets nefastes des changements climatiques.

\section{References :}

1. Briquet, J.P., Mahe, G., \& Bamba, F. (1996). Changements climatiques récents et modification du régime hydrologique du fleuve Niger à Koulikoro (Mali). L'hydrologie tropicale: géoscience et outil pour le développement, Actes de la conférence de Paris, mai 1995, IAHS Publ. no. 238, p. 157-166.

2. CNUCED (2010). L'agriculture à la croisée des chemins: sécurité alimentaire et changements climatiques, $\mathrm{N}^{\circ} 18$.

3. Direction Nationale de la Météorologie (2007). Programme d'Action National d'Adaptation aux Changements Climatiques : 100 p.

4. Fundation DARA International (2010). Climate Vulnerability Monitor 2010 - The State of the Climate Crisis. Editor : DARA, 286 p.

5. GIEC (2014). Changements climatiques 2014: Rapport de synthèse. Contribution des Groupes de travail I, II et III au cinquième Rapport d'évaluation du Groupe d'experts intergouvernemental sur l'évolution du climat [Sous la direction de l'équipe de rédaction principale, R.K. Pachauri et L.A. Meyer]. GIEC, Genève, Suisse, 161 p.

6. Kouassi, A., Assamoi, P., Bigot, S., Diawara, A., Schayes, G., Yoroba, F., \& Kouassi, B. (2010). Étude du climat ouest-africain à l'aide du modèle atmosphérique régional M.A.R., Climatologie, p. 39-55.

7. Kouassi, A.M., Kouame, K.F., Koffi, Y.B., Dje, K.B., Paturel, J.E., \& Oulare, S. (2010). Analyse de la variabilité climatique et de ses influences sur les régimes pluviométriques saisonniers en Afrique de l'Ouest : cas du bassin versant du N'zi (Bandama) en Côte d'Ivoire, Cybergeo : European Journal of Geography [En ligne], Environnement, Nature, Paysage, document 513, mis en ligne le 07 décembre 2010, consulté le 16 mars 2018. URL : http://journals.openedition.org/cybergeo/23388 ; DOI : 10.4000/cybergeo.23388

8. Le Barbe, L., Lebel, T., \& Tapsoba, D. (2001). Rainfall variability in West Africa during the years 1950-1990, Journal of Climate, p. 187202.

9. L'hôte, Y., Dubreuil, P., \& Lerique, J. (1996). Carte des types de climats « en Afrique Noire à l'ouest du Congo ». Rappels, et extension aux régimes hydrologiques. L'hydrologie tropicale: géoscience et outil pour le développement (Actes de la conférence de Paris, mai 1995). IAHS Publ. no. 238, p. 55-65. 
10. Lobanov, V.A., Smirnov, I.A., \& Shadursky, A.E. (2011). Laboratory workshop on climatology, Part I, Textbook, St. Petersburg, RSHU Publishers, $134 \mathrm{p}$.

11. Nicholson, S.E. \& Webster, P.J. (2007). A physical basis for the interannual variability of rainfall in the Sahel. Quarterly Journal of the Royal Meteorological Society, 133:2065-2084.

12. OMM (2011). Guide des pratiques climatologiques, $\mathrm{N}^{\circ} 100$, Édition 2011.

13. Ouedraogo, M. (2001). Contribution à l'étude de l'impact de la variabilité climatique sur les ressources en eau en Afrique de l'Ouest. Analyse des conséquences d'une sécheresse persistante : normes hydrologiques et modélisation régionale, Thèse de Doctorat, Université de Montpellier II, France, 258p.

14. Paturel, J.E., Servat, E., Delattre, M.O., \& Lubes-Niel, H. (1998). Analyse de séries pluviométriques de longue durée en Afrique de l'Ouest et Centrale non sahélienne dans un contexte de variabilité climatique, Hydrological Sciences Journal, 43:6, 937-946.

15. Roudier, P. (2012). Climat et agriculture en Afrique de l'Ouest: Quantification de l'impact du changement climatique sur les rendements et évaluation de l'utilité des prévisions saisonnières, Thèse de doctorat, Ecole des Hautes Etudes en Sciences Sociales (EHESS), Paris, France, 189p.

16. Roudier, P. \& Quirion, P. (Juin 2009). Bilan des changements climatiques passés et futurs au Mali : rapport pour Action Contre la Faim. Centre International de Recherche sur l'Environnement et le Développement (CIRED), $42 \mathrm{p}$. 